\title{
Numerical Estimation Method of Orthotropic Material Properties of a Roving for Reinforcement of Composite Materials
}

\author{
Gergely Bojtár ${ }^{1}$, Béla M. Csizmadia ${ }^{2}$, János Égert ${ }^{1}$ \\ ${ }^{1}$ Széchenyi István University, Department of Applied Mechanics, \\ 9026 Győr, Egyetem tér 1, Hungary, bojtar@sze.hu, egert@sze.hu \\ ${ }^{2}$ Szent István University, Institute of Mechanics and Machinery, \\ 2103 Gödöllő, Páter K. u. 1, Hungary, csizmadia.bela@gek.szie.hu
}

Abstract: Knowledge of the material properties is important at machine design from composite materials. This information cannot be found in related literature and is not standard, therefore, it was necessary to determine, by measurements and by basic experiments of the mechanics of materials. This paper presents a new $3 D$ finite element (FE) model-cell for the modeling of the material properties of a roving. By this numerical modeling method, one can determine the orthotropic and macroscopic material properties of a roving. This model-cell, models numerically, a roving, which consists of multiples of thousands of fibers that are embedded in a matrix material, as a homogenized orthotropic material. The numerical results of the material properties of the roving, can be applied to the definition of the macroscopic material properties of fiber reinforced composite laminates.

Keywords: roving; orthotropic material properties; fiber reinforced composite; finite element method; model-cell

\section{Introduction}

Fiber reinforced composite is a plastic material which is reinforced by glass, carbon, aramid, etc., fibers or roving's. Textile composite plates are layer structured and each layer contains a textile which is impregnated by some sort of plastic. The fibers in the textile are ordered usually in roving's. In a roving there are many thousand straight fibers laying parallel to each other or running in a twisted form and also between the fibers there is plastic material [2]. The diameter of the fibers can be measured in $\mu \mathrm{m}-$, the thickness of a textile and a composite layer can be measured in tenth of mm-s. The plastic in the composite, the so called matrix, is the carrier or embedding material for the fibers, which can be e.g. a thermosoftening or thermosetting polymer. 
For dimensioning or stress checking of a composite structure one needs the material properties of the composite plate. This can be defined by measurements and this is the usual way nowadays [1]. By knowing the textile and the matrix, which was chosen based on an earlier experience, test specimens are produced and the material properties are determined by measurement. There are numerical methods as well for the calculation of material properties of the composite plate, assuming that we know the material properties of the layers [3]. However, the material properties of the layers are unknown in general either. For their definition there is a finite element computation method for building up the material properties of composite layer from the material properties of the textile and the matrix. These numerical methods deliver an approach for the properties of layers, but these calculations are not accurate enough $[7,11,12]$. Failures and uncertainties occur at requirements of the boundary conditions.

The ultimate goal of this research is to determine or at least to estimate numerically, the macroscopic material properties of a textile composite layer with a known geometry and material with a finite element (FE) model-cell. Then, this numerical result is compared to the results of the measurement. But before we would be able to solve this problem, which will be presented in another following article, first we need to understand the behavior of the materials building up the composite layer. The matrix can be described as a homogenous isotropic material, therefore its material properties can be determined safely, by measurement, regardless of the actual composite. The orthotropic material properties resulting from the material structures of the fibers that build up the roving can be assumed known from factory catalogues or literature. These are not modeled separately but we consider the roving itself as a "fiber" in the layer model-cell. The roving can be very multifarious and, as we mentioned earlier, impregnated by matrix material. This means that one roving should be considered as a structure and on a macroscopic level its linear elastic orthotropic material properties should be determined by a computational method. This is our aim with the present paper.

The constitutive equation of linear elastic orthotropic material can be described by [2] to (1) relationship:

$$
\left[\begin{array}{c}
\bar{\varepsilon}_{r 1} \\
\bar{\varepsilon}_{r 2} \\
\bar{\varepsilon}_{r 3} \\
\bar{\gamma}_{r 12} \\
\bar{\gamma}_{r 23} \\
\bar{\gamma}_{r 13}
\end{array}\right]=\left[\begin{array}{cccccc}
\frac{1}{E_{r 1}} & -\frac{v_{r 21}}{E_{r 2}} & -\frac{v_{r 31}}{E_{r 3}} & 0 & 0 & 0 \\
-\frac{v_{r 12}}{E_{r 1}} & \frac{1}{E_{r 2}} & -\frac{v_{r 32}}{E_{r 3}} & 0 & 0 & 0 \\
-\frac{v_{r 13}}{E_{r 1}} & -\frac{v_{r 23}}{E_{r 2}} & \frac{1}{E_{r 3}} & 0 & 0 & 0 \\
0 & 0 & 0 & \frac{1}{G_{r 12}} & 0 & 0 \\
0 & 0 & 0 & 0 & \frac{1}{G_{r 23}} & 0 \\
0 & 0 & 0 & 0 & 0 & \frac{1}{G_{r 13}}
\end{array}\right]\left[\begin{array}{c}
\bar{\sigma}_{r 1} \\
\bar{\sigma}_{r 2} \\
\bar{\sigma}_{r 3} \\
\bar{\tau}_{r 12} \\
\bar{\tau}_{r 23} \\
\bar{\tau}_{r 13}
\end{array}\right] .
$$


The matrix of material properties in (1) is symmetric, therefore the relationships between Young's modulus and Poisson's ratio look as follows [2]:

$\frac{v_{r 12}}{E_{r 1}}=\frac{v_{r 21}}{E_{r 2}}, \quad \frac{v_{r 13}}{E_{r 1}}=\frac{v_{r 31}}{E_{r 2}}, \quad \frac{v_{r 23}}{E_{r 2}}=\frac{v_{r 32}}{E_{r 3}}$.

Considering formula (2) the matrix of material properties in equation (1) has nine independent material constants:

$E_{r 1}, E_{r 2}, E_{r 3}, v_{r 12}, v_{r 23}, v_{r 13}, G_{r 12}, G_{r 23}, G_{r 13}$.

For the roving model-cell with Volume $V$, the average strain and stress can be introduced and defined as follows [7], [8], [11]:

$\bar{\varepsilon}_{r i j}=\frac{1}{V} \int_{(V)} \varepsilon_{r i j} d V$

$\bar{\sigma}_{r i j}=\frac{1}{V} \int_{(V)} \sigma_{r i j} d V$

\section{The Roving Model-Cell}

The aim of creation of the roving model-cell is to be able to model the roving as a homogeneous material. This can be reached by considering average strains and stresses according to (3) and (4). The complete roving can be modeled as homogeneous population of such model-cells.

\subsection{The Geometry and Finite Element Mesh of the Roving Model-Cell}

The reinforcing fibers in the roving are not regularly positioned in the matrix. In Figure 1 we can see the microscopic structure of a carbon/epoxy composite roving, where the diameter of a fiber is $7 \mu \mathrm{m}$ [9].

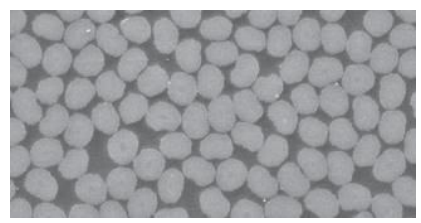

Figure 1

The real position of the fibers in the roving, in the matrix 
It is also necessary to model the position of the fibers in the matrix [6]. At the modeling is assumed that the reinforcing fibers are positioned in the model-cell in a regular hexagonal shape (Figure 2). This is a good geometrical approach (Figure 3 ) to the real case illustrated in Figure 1.

In order to have more simple boundary conditions we configure the side surfaces of the model-cell parallel to the planes of the principal material-directions [5]. At a hexagonal shape there are two possible roving model-cell configurations (Figure 3 ). For our further examination, we chose the geometry on the right side. The ratio of two sides of these model-cells is:

$$
\frac{a}{b}=\frac{1}{\sqrt{3}} \text {. }
$$

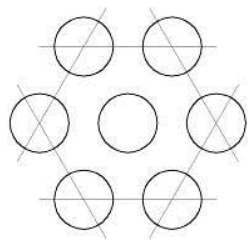

Figure 2

Regular hexagonal shape

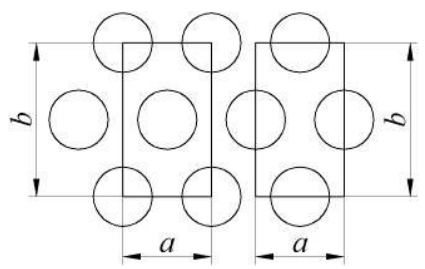

Figure 3

Geometry of possible roving model-cells

The usual ideal cross-section of an untwisted, straight roving is a rectangle ending up in a semicircle, an ellipse or a lens shape (Figure 4) [11]. The cross-section area of the roving is $A_{r}$, where $n_{f}$ number of fiber is located with diameter $d_{f}$. This area is provided by the geometry of the roving.

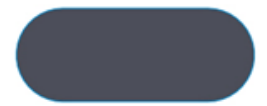

a) Rectangular shape ending up in a semicircle

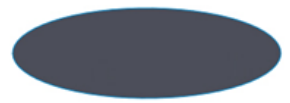

b) Ellipse shape

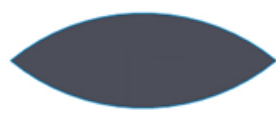

c) Lens shape

Figure 4

The usual cross-sections of an untwisted straight roving

The volume ratio of the fibers in the roving can be calculated from the crosssection area of the fibers and from the whole area of the roving:

$\varphi_{f r}=\frac{A_{f r}}{A_{r}}=\frac{d_{f}^{2} \pi}{4 A_{r}} n_{f}$.

It is necessary to determine this in order to have the same ratio in the roving model-cell. 
The dimensions of the roving model-cell are $10^{3}$ times larger that of the dimensions of the real roving. The units used at the real roving and the roving modelcell are summarized in Table 1. In order to have stresses in the model-cell that are identical to the stresses in the real roving it is necessary to apply force on the model-cell which is $10^{6}$ times higher that of the real force (Table 1).

Table 1

Units in reality and in the roving model-cell

\begin{tabular}{|l|c|l|}
\hline & Real roving & Roving model-cell \\
\hline Length: $l$ & $\mu \mathrm{m}$ & $\mathrm{mm}=\mu \mathrm{m} \cdot 10^{3}$ \\
\hline Area: $A$ & $\mu \mathrm{m}^{2}$ & $\mathrm{~mm}^{2}=\mu \mathrm{m}^{2} \cdot 10^{6}$ \\
\hline Force: $F$ & $\mu \mathrm{N}$ & $\mathrm{N}=\mu \mathrm{N} \cdot 10^{6}$ \\
\hline Stresses: $\sigma_{i}, \tau_{i j}$ & $\frac{\mu \mathrm{N}}{\mu \mathrm{m}^{2}}=\frac{10^{-6} \mathrm{~N}}{10^{-6} \mathrm{~mm}^{2}}=\mathrm{MPa}$ & $\frac{\mathrm{N}}{\mathrm{mm}^{2}}=\mathrm{MPa}$ \\
\hline
\end{tabular}

The roving model-cell (Figure 3) includes altogether two fibers. Considering the volume ratio (6) and the side ratio (5) the $a$ and $b$ side length of the roving modelcell can be calculated for the creation of the model-cell of the given roving:

$a=\sqrt{\frac{d_{f}^{2} \pi}{2 \sqrt{3} \varphi_{f r}},} \quad b=\sqrt{3} a$.

Figure 5 shows also the identification signs of the six side-surfaces of the cell. The dimension of the side-areas is clear.

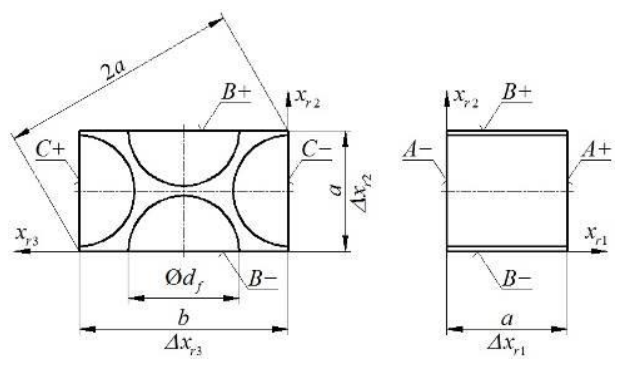

Figure 5

Dimensions of the roving model-cell used for the numerical modeling

A next paper will show a textile layer composite model-cell as well. The results of this model-cell will be checked by experiments. Roving with cross section shown in Figure 6 are applied in this model-cell of the textile layer (see also: Section 2.5). 


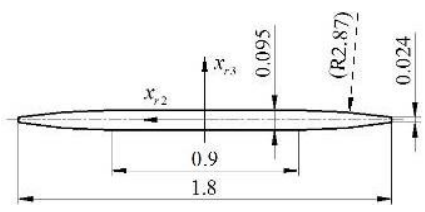

Figure 6

The cross section of the applied roving in the textile layer model-cell

The dimensions of the applied roving in the examined textile material:

$$
d_{f}=7 \mu \mathrm{m}, n_{f}=3000 \mathrm{db}, \varphi_{f r}=0.7667 .
$$

The dimensions of the examined roving model-cell according to the Table 1:

$$
\begin{aligned}
& d_{f}=7 \mathrm{~mm}, \quad a=7.613 \mathrm{~mm}, \quad b=13.186 \mathrm{~mm}, \quad \varphi_{f r}=0.7667, \\
& A_{A+}=A_{A-}=A_{B+}=A_{B-}=100.385 \mathrm{~mm}^{2}, A_{C+}=A_{C-}=57.958 \mathrm{~mm}^{2} .
\end{aligned}
$$

The finite element computations were carried out with the NX I-deas 6.1 program system. The material coordinate system of the roving is $x_{r 1} x_{r 2} x_{r 3}$, where $x_{r 1}$ is the direction parallel to the single fibers (Figure 5). Due to the periodicity we need to apply the so called periodical boundary conditions for the roving model-cell. Therefore we need to generate the finite element mesh in the way that, on the opposite surfaces there should be nodes at the same coordinates and so node-pairs shall be created in the model-cell. A mesh consisting of quadratic hexahedron elements was generated for the examined roving model-cell (Figure 8). The geometry of the model-cell was split into, consisting of five larger volume sections (Figure 8), into further 80 sections in order to be able to create a regular net (Figure 7). In Figure 8 the finite element mesh of the roving model-cell is seen, which consists of 34994 hexahedron elements and 149521 nodes.

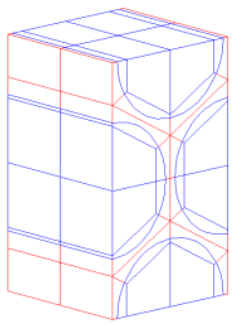

Figure 7

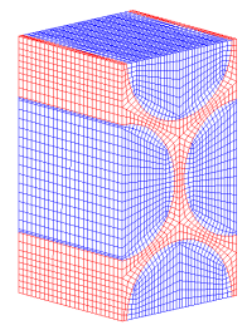

Figure 8

Volume sections of the roving model-cell

Due to the regular hexagonal order of the reinforcing fibers the material properties are identical in $x_{r 2}, x_{r 3}$ direction and in $x_{r 1} x_{r 2}, x_{r 1} x_{r 3}$ planes:

$$
E_{r 2}=E_{r 3}, v_{r 12}=v_{r 13}, G_{r 12}=G_{r 13} .
$$




\subsection{The Loading of the Roving Model-Cell}

In consideration of the relationships (8) it is enough to model two axial tensions and two plane shears with the roving model-cell.

To ensure the periodicity we prescribed a kinematic loading is prescribed, i.e. a node displacement field for the roving model cell. The nodes at the vertex points of the model-cell and at the center points of the side surfaces are identified with node numbers (Figure 9).
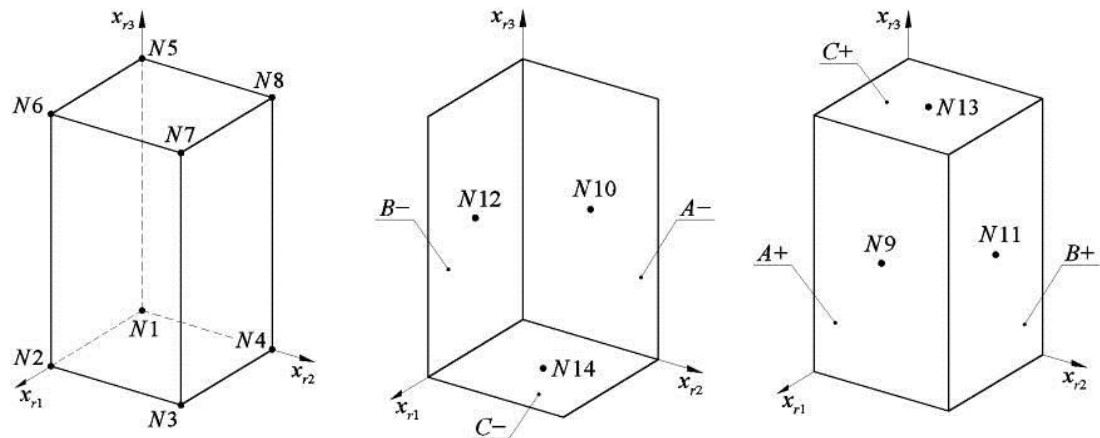

Figure 9

Notation of the nodes at the eight vertexes and at the six center points of the sides of the model-cell

The node displacement vector for the roving model-cell in the $x_{r 1} x_{r 2} x_{r 3}$ coordinate system:

$\vec{u}=u \vec{e}_{r 1}+v \vec{e}_{r 2}+w \vec{e}_{r 3}$.

For the roving model-cell (Figures 7 and 8 ) the following general boundary conditions can be applied [7]:

$u_{i}^{j+}\left(x_{1}, x_{2}, x_{3}\right)-u_{i}^{j-}\left(x_{1}, x_{2}, x_{3}\right)=\Delta x_{r j} \bar{\varepsilon}_{r i j}=c_{i}^{j}$.

On the surface pair perpendicular to $x_{r j}$ axis $u_{i}^{j+}, u_{i}^{j-}$ are displacements in $x_{i}$ direction the $x_{1} x_{2} x_{3}$ global coordinate system. The $j+$ index indicates the positive direction of $x_{r j}$ axis whereas the $j$ - indicates the negative direction of $x_{r j}$ (Figure 5). $\Delta x_{r j}$ is the side length of the roving model-cell. $c_{i}^{j}(i=j=1,2,3)$ is the change in $x_{r j}$ directions of distance (displacement difference) of the side surface pairs of the model cell, whereas $c_{i}^{j}=c_{j}^{i}(i \neq j=1,2,3)$ is the displacement difference resulting from the shear of the side surfaces. The (10) boundary conditions ensure the periodicity and the continuity of the displacement field in the roving of composite material. Equation (10) provides the displacement differences between the proper nodes on both opposite surfaces. $u_{i}^{j+}, u_{i}^{j-}$ displacements are 
the functions of $x_{1}, x_{2}, x_{3}$ coordinates, therefore these surfaces not necessarily stay in plane during the deformation. $c_{i}^{j}$ are prescribed displacement differences or computed by the finite element method.
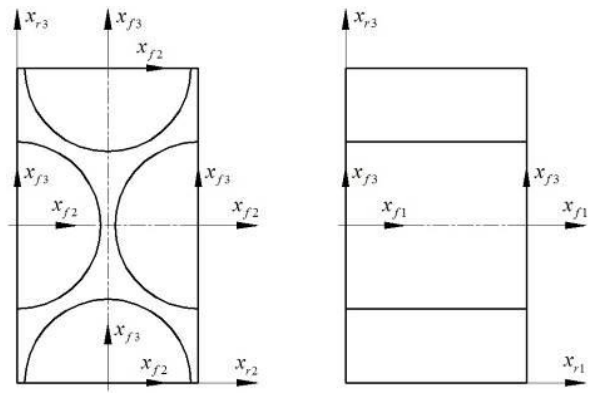

Figure 10

$x_{r 1} x_{r 2} x_{r 3}$ material coordinate system of the roving model-cell and $x_{f 1} x_{f 2} x_{f 3}$ material coordinate system of the reinforcing fibers

In Figure 10 the $x_{r 1} x_{r 2} x_{r 3}$ material coordinate system of the roving model cell and the $x_{f 1} x_{f 2} x_{f 3}$ material coordinate system of the reinforcing fibers are seen. At the fibers $x_{f 1} x_{f 2}$ and $x_{f 1} x_{f 3}$ are planes of symmetry. Due to the fact that the side surfaces of the roving model cell are identical to the surfaces of $x_{f 1} x_{f 2}, x_{f 2} x_{f 3}$ and $x_{f 1} x_{f 3}$ of the fibers, at the tensile-test, illustrated below, the side surfaces of the model cell remain plane and displace parallel with the surfaces of the main material directions. On the other hand there is no deformation at the shear test in the $x_{r i} x_{r j}$ surface, on the side surfaces perpendicular to $x_{r i}$ axis in $x_{r j}$ direction and on the side surfaces perpendicular to $x_{r j}$ axis in $x_{r i}$ direction.

As mentioned earlier, with the introduction of the roving model-cell, we can model the roving as a homogenous material, and we can also determine, by basic experiments of strength of materials (by tensile-compression tests and shear tests), the material properties. With the instruction of kinematic loading, we will give the displacement for whole side surfaces of model-cell instead of certain selected nodes. From these kinematic loadings reaction forces occur on the side surfaces. We reduced the side surface reaction force to the center node of the side in a way that we prescribed the displacement to the central node and coupled all the nodes on the surface in the given direction. This prescription ensures that the side surface can displace as a rigid plane in the prescribed direction. Due to the fact that the side surfaces of the roving model-cell coincide to the surfaces of the material main directions of the fibers, the average of the model-cell appears on the side surfaces. The average stress is determined by the fraction of the reaction force appearing on the side surface and the area of the side surface: 
$\bar{\sigma}_{r i j}=\frac{1}{A_{j}} \int_{\left(A_{j}\right)} \sigma_{r i j} d A=\frac{F_{r i}}{A_{j}}$.

$F_{r i}$ is a reaction force in $x_{r i}$ direction and it is appearing on a side surface $A_{j}$ area that is perpendicular to $x_{r j}$ axis.

\subsection{Tensile-Compression Test Simulation with the Roving Model-Cell}

For computation of $E_{r 1}$ Young's modulus and $v_{r 12}, v_{r 13}$ Poisson's ratios of the roving and for determination of $E_{r 2}, v_{r 21}, v_{r 23}$ we need to simulate a pure tension-compression on the roving model-cell in $x_{r 1}$ direction and $x_{r 2}$ direction respectively. For the calculation of $E_{r 3}, v_{r 31}, v_{r 32}$ we need to simulate a tension in $x_{r 3}$ direction. Since the reinforcing fibers are in a regular hexagonal order, the material properties are the same in $x_{r 2}$ and $x_{r 3}$ directions. Because of the numerical verification we also simulated a tension in $x_{r 3}$ direction but we will not give the details of that here, we will only show the test simulation in $x_{r 1}$ and $x_{r 2}$ directions.

In the strain matrix in (1) only three tensile strains are different from zero, the three coordinates of the axial tensions. $\left(\bar{\varepsilon}_{r 1}, \bar{\varepsilon}_{r 2}, \bar{\varepsilon}_{r 3} \neq 0\right)$. At tension (10) formula looks as follows:

$$
c_{i}^{j}=\Delta x_{r j} \bar{\varepsilon}_{r j}, \quad \quad \bar{\varepsilon}_{r j}=\frac{c_{i}^{j}}{\Delta x_{r j}}, \quad(i=j=1,2,3) .
$$

The condition of parallelism at the tensile loading is ensured by coupling all the nodes on the side surface in direction perpendicular to the surface. These requirements are summarized by the (13) - (15) relationships. According to (13) equation we couple all nodes on $A+$ surface in $x_{r 1}$ direction. The independent node is $N 2$ and that means the displacements of all nodes on $A+$ surface are identical with the displacement of $N 2$ node in $x_{r 1}$ direction.

$$
\begin{array}{ll}
u_{A-}=u\left(0 ; x_{r 2} ; x_{r 3}\right)=u_{N 1}, & u_{A+}=u\left(a ; x_{r 2} ; x_{r 3}\right)=u_{N 2}, \\
v_{B-}=v\left(x_{r 1} ; 0 ; x_{r 3}\right)=v_{N 5}, & v_{B+}=v\left(x_{r 1} ; a ; x_{r 3}\right)=v_{N 8}, \\
w_{C-}=w\left(x_{r 1} ; x_{r 2} ; 0\right)=w_{N 3}, & w_{C+}=w\left(x_{r 1} ; x_{r 2} ; b\right)=w_{N 7} .
\end{array}
$$

In (13) - (15) relationships:

$$
0 \leq x_{r 1} \leq a, \quad 0 \leq x_{r 2} \leq a, \quad 0 \leq x_{r 3} \leq b .
$$


At the $x_{r 1}$ and $x_{r 2}$ directional tensions we clamped the $A-, B$ - and $C$ - side surfaces at their central node in direction perpendicular to the surface:

$u_{A-}=u_{N 10}=0, \quad v_{B-}=v_{N 12}=0, \quad w_{C-}=w_{N 14}=0$.

The kinematic loading belonging to both axial tension tests of the roving modelcell is defined as follows: at the $x_{r j}$ directional, axial tension we gave the $\bar{\varepsilon}_{r j}$ tensile strain of the model-cell, and we simulate by FEM the normal displacement of the side surface with $x_{r j}$ normal direction to (12). We did not give the transversal contraction of the surface, we computed it numerically.

At $x_{r 1}$ directional tension $\bar{\varepsilon}_{r 1}$ is given, from which the displacement of the $A+$ side surface is:

$u_{A+}=u_{N 9}=a \bar{\varepsilon}_{r 1}$.

At $x_{r 2}$ directional tension $\bar{\varepsilon}_{r 2}$ is given, from which:

$v_{B+}=v_{N 11}=a \bar{\varepsilon}_{r 2}$.

At tension, in both loading case, we still need to ensure that the opposite nodes displace in same way in the plane on the side surface. The node pairs should be coupled on the opposite side surfaces, except the edges of the model-cell, as follows:

$$
\begin{aligned}
& A-/ A+: \quad v\left(0 ; x_{r 2} ; x_{r 3}\right)=v\left(a ; x_{r 2} ; x_{r 3}\right), \quad w\left(0 ; x_{r 2} ; x_{r 3}\right)=w\left(a ; x_{r 2} ; x_{r 3}\right), \\
& B-/ B+: \quad u\left(x_{r 1} ; 0 ; x_{r 3}\right)=u\left(x_{r 1} ; a ; x_{r 3}\right), \quad w\left(x_{r 1} ; 0 ; x_{r 3}\right)=w\left(x_{r 1} ; a ; x_{r 3}\right), \\
& C-/ C+: \quad u\left(x_{r 1} ; x_{r 2} ; 0\right)=u\left(x_{r 1} ; x_{r 2} ; b\right), \quad v\left(x_{r 1} ; x_{r 2} ; 0\right)=v\left(x_{r 1} ; x_{r 2} ; b\right) \text {. }
\end{aligned}
$$

In (20) - (22) and (24) - (26) relationships:

$$
0<x_{r 1}<a, \quad 0<x_{r 2}<a, \quad 0<x_{r 3}<b .
$$

We need to couple the node pairs at the opposite edges, except the corner points of the model-cell, according to (24) - (26) relationships:

For edges in $x_{r 1}$ direction:

$$
u\left(x_{r 1} ; 0 ; 0\right)=u\left(x_{r 1} ; a ; 0\right)=u\left(x_{r 1} ; a ; b\right)=u\left(x_{r 1} ; 0 ; b\right),
$$

For edges in $x_{r 2}$ direction:

$$
v\left(0 ; x_{r 2} ; 0\right)=v\left(0 ; x_{r 2} ; b\right)=v\left(a ; x_{r 2} ; b\right)=v\left(a ; x_{r 2} ; 0\right),
$$

For edges in $x_{r 3}$ direction:

$$
w\left(0 ; 0 ; x_{r 3}\right)=w\left(a ; 0 ; x_{r 3}\right)=w\left(a ; a ; x_{r 3}\right)=w\left(0 ; a ; x_{r 3}\right)
$$


Figure 11 shows the coupling of the nodes between the opposite side surfaces and the edges. It is necessary to skip the edges at the opposite sides and the corners at the opposite edges so that the model-cell will not be overdetermined.
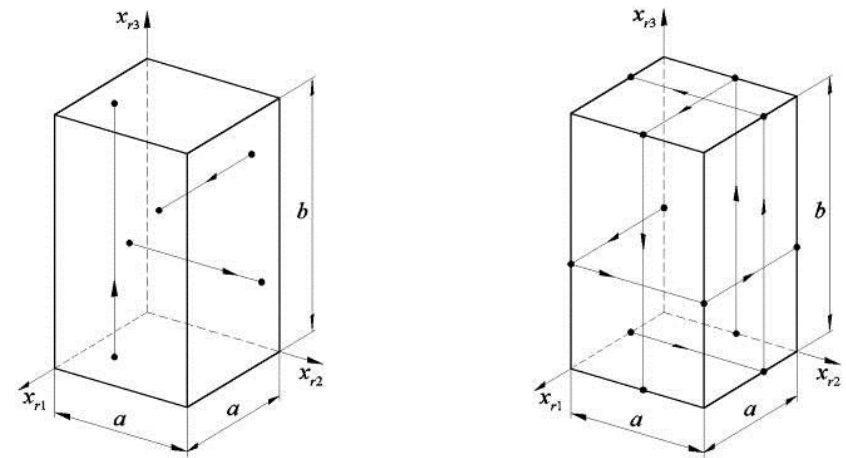

Figure 11

Coupling between the nodes

Table 2 summarizes the numerically determined reaction forces with the roving model-cell for both axial tensions as well as the displacement field (cross contraction) perpendicular to the direction of the tension.

Table 2

Properties defined by the finite element model-cell in case of both load-cases

\begin{tabular}{|l|l|c|}
\hline & Reaction force on the side surfaces & Cross contraction \\
\hline Tension in $x_{r 1}$ direction & $\vec{F}_{r A+}=-\vec{F}_{r A-}, \vec{F}_{r B-}=\vec{F}_{r C-}=\overrightarrow{0}$ & $v_{B+}, w_{C+}$ \\
\hline Tension in $x_{r 2}$ direction & $\vec{F}_{r B+}=-\vec{F}_{r B-}, \vec{F}_{r A-}=\vec{F}_{r C-}=\overrightarrow{0}$ & $u_{A+}, w_{C+}$ \\
\hline
\end{tabular}

At $x_{r 1}$ directional tension we calculate the average $\bar{\sigma}_{r 1}$ normal stress from the reaction force created on $A+$ side surface and from the area of the side surface according to (11), from which we can determine the Young's modulus in the usual way. Poisson's ratio can also be calculated in the usual way from the relevant data received from the calculated displacement field. We can also follow these methods for the cross directions of the roving model-cell.

Material properties determined above of a single roving cannot be measured directly. Therefore the validity of the proposed model-cell can only be verified directly by measurement on a composite specimen. For this we need to create the model-cell for a textile composite layer which we will discuss in our next paper. Applying this layer model-cell, using the results generated by the roving modelcell we can verify only together the validity and accuracy of both model-cells. We have given the geometrical and material properties of the composite specimen, used for the verification of model-cells in Section 2.5. Table 3 summarizes the results (for the two axial tensions) generated by numerical methods with these 
data. The table consists of the kinematic loading, the reaction forces determined numerically, the cross contractions (displacements) and the average stresses and the material properties computed from those for both tensions. We publish these as application of the roving model-cell and use them at the evaluation of the measurements.

Table 3, Part 1

The given data and determined material properties for the applied roving model-cell

\begin{tabular}{|l|l|}
\hline \multicolumn{2}{|c|}{ Tension in $x_{r 1}$ direction } \\
\hline Kinematic loading & $\bar{\varepsilon}_{r 1}=2.5 \cdot 10^{-3}, u_{A+}=u_{N 9}=19.0325 \cdot 10^{-3} \mathrm{~mm}$ \\
\hline \multirow{2}{*}{$\begin{array}{l}\text { Numerically determined } \\
\text { quantities }\end{array}$} & $\vec{F}_{r A+}=\left(44479.2 \vec{e}_{r 1}\right) \mathrm{N}, \vec{F}_{r A-}=\left(-44479.2 \vec{e}_{r 1}\right) \mathrm{N}$ \\
\cline { 2 - 3 } & $v_{B+}=-3.854 \cdot 10^{-3} \mathrm{~mm}, w_{C+}=-6.675 \cdot 10^{-3} \mathrm{~mm}$ \\
\hline Average stress & $\bar{\sigma}_{r 1}=443.09 \mathrm{MPa}$ \\
\hline Material properties & $E_{r 1}=177236 \mathrm{MPa}, v_{r 12}=0.202, v_{r 13}=0.202$ \\
\hline
\end{tabular}

Table 3, Part 2

The given data and determined material properties for the applied roving model-cell

\begin{tabular}{|l|l|}
\hline \multicolumn{2}{|c|}{ Tension in $x_{r 2}$ direction } \\
\hline Kinematic loading & $\bar{\varepsilon}_{r 2}=2.5 \cdot 10^{-3}, v_{B+}=v_{N 11}=19.0325 \cdot 10^{-3} \mathrm{~mm}$ \\
\hline \multirow{2}{*}{$\begin{array}{l}\text { Numerically determined } \\
\text { quantities }\end{array}$} & $\vec{F}_{r B+}=\left(2597.9 \vec{e}_{r 2}\right) \mathrm{N}, \vec{F}_{r B-}=\left(-2597.9 \vec{e}_{r 2}\right) \mathrm{N}$ \\
\cline { 2 - 2 } & $u_{A+}=-2.251 \cdot 10^{-4} \mathrm{~mm}, w_{C+}=-14.189 \cdot 10^{-3} \mathrm{~mm}$ \\
\hline Average stress & $\bar{\sigma}_{r 2}=25.88 \mathrm{MPa}$ \\
\hline Material properties & $E_{r 2}=10352 \mathrm{MPa}, v_{r 21}=0.012, v_{r 23}=0.430$ \\
\hline
\end{tabular}

\subsection{Shear Test Simulation with the Roving Model-Cell}

According to (8): $G_{r 12}=G_{r 13}$. Because of numerical verification we modeled the shear test on both surfaces of roving model-cell, however, we will not discuss the details here. At the shear on $x_{r i} x_{r j}$ surface $\bar{\gamma}_{r i j} \neq 0$ and the other strain coordinates are zeroes in the strain tensor. Also at the shear we prescribed the kinematic loading and here $\bar{\gamma}_{r i j}$ is the average shearing strain. We simulated pure shear with the roving model-cell. From (10) follows for the pure shear:

$$
c_{i}^{j}=\Delta x_{r j} \frac{1}{2} \bar{\gamma}_{r i j}, \quad \quad c_{j}^{i}=\Delta x_{r i} \frac{1}{2} \bar{\gamma}_{r j i} \quad(i \neq j=1,2,3) .
$$


For determination of the shear modulus $G_{r 12}$ of roving material we need to model pure shear on the $x_{r 1} x_{r 2}$ surface. The given shearing strain is:

$\frac{1}{2} \bar{\gamma}_{r 12}=\frac{1}{2} \bar{\gamma}_{r 21}, \quad \bar{\gamma}_{r 12}=\frac{1}{2} \bar{\gamma}_{r 12}+\frac{1}{2} \bar{\gamma}_{r 21}$.

The pure shear is simulated by a given displacement in $x_{r 2}$ direction on the side surfaces perpendicular to the $x_{r 1}$ axis and by a given displacement in $x_{r 1}$ direction on the side surfaces perpendicular to the $x_{r 2}$ axis of the roving model-cell. At the shear on $x_{r 1} x_{r 2}$ plane the $C+$ and $C$ - side surfaces remain planes and do not displace in $x_{r 3}$ direction. We have given the above kinematic requirements (27) by using the given values for the nodes at the center points of the side surfaces, as follows:

$$
\begin{array}{ll}
v_{A-}=v_{N 10}=0, & v_{A+}=v_{N 9}=a \frac{1}{2} \bar{\gamma}_{r 21}, \\
u_{B-}=u_{N 12}=0, & u_{B+}=u_{N 11}=a \frac{1}{2} \bar{\gamma}_{r 12}, \\
w_{C-}=w_{N 14}=0, & w_{C+}=w_{N 13}=0 .
\end{array}
$$

At the shear modeling we need to couple the nodes on the side surfaces as follows:

$$
\begin{array}{ll}
v_{A-}=v\left(0 ; x_{r 2} ; x_{r 3}\right)=v_{N 1}, & v_{A+}=v\left(a ; x_{r 2} ; x_{r 3}\right)=v_{N 2}, \\
u_{B-}=u\left(x_{r 1} ; 0 ; x_{r 3}\right)=u_{N 5}, & u_{B+}=u\left(x_{r 1} ; a ; x_{r 3}\right)=u_{N 8}, \\
w_{C-}=w\left(x_{r 1} ; x_{r 2} ; 0\right)=w_{N 3}, & w_{C+}=w\left(x_{r 1} ; x_{r 2} ; b\right)=w_{N 7} .
\end{array}
$$

In relation $(32)-(34)$ :

$0 \leq x_{r 1} \leq a, \quad 0 \leq x_{r 2} \leq a, \quad 0 \leq x_{r 3} \leq b$.

The node pairs should be coupled at the opposite side surfaces, with exception of the edges of the model cell, according to (36) - (38):

$$
\begin{aligned}
& A-/ A+: \quad u\left(0 ; x_{r 2} ; x_{r 3}\right)=u\left(a ; x_{r 2} ; x_{r 3}\right), \quad w\left(0 ; x_{r 2} ; x_{r 3}\right)=w\left(a ; x_{r 2} ; x_{r 3}\right), \\
& B-/ B+: \quad v\left(x_{r 1} ; 0 ; x_{r 3}\right)=v\left(x_{r 1} ; a ; x_{r 3}\right), \quad w\left(x_{r 1} ; 0 ; x_{r 3}\right)=w\left(x_{r 1} ; a ; x_{r 3}\right), \\
& C-/ C+: \quad u\left(x_{r 1} ; x_{r 2} ; 0\right)=u\left(x_{r 1} ; x_{r 2} ; b\right), \quad v\left(x_{r 1} ; x_{r 2} ; 0\right)=v\left(x_{r 1} ; x_{r 2} ; b\right) .
\end{aligned}
$$

In relations (36) - (38) and (40) - (42):

$0<x_{r 1}<a, \quad 0<x_{r 2}<a, \quad 0<x_{r 3}<b$. 
At the opposite edges, with exception of the corner points of the model cell, we need to couple the node pairs as follows:

For edges in $x_{r 1}$ direction:

$$
v\left(x_{r 1} ; 0 ; 0\right)=v\left(x_{r 1} ; a ; 0\right)=v\left(x_{r 1} ; a ; b\right)=v\left(x_{r 1} ; 0 ; b\right),
$$

For edges in $x_{r 2}$ direction:

$$
u\left(0 ; x_{r 2} ; 0\right)=u\left(0 ; x_{r 2} ; b\right)=u\left(a ; x_{r 2} ; b\right)=u\left(a ; x_{r 2} ; 0\right),
$$

For edges in $x_{r 3}$ direction:

$$
w\left(0 ; 0 ; x_{r 3}\right)=w\left(a ; 0 ; x_{r 3}\right)=w\left(a ; a ; x_{r 3}\right)=w\left(0 ; a ; x_{r 3}\right) .
$$

With the help of the roving model cell, the reaction forces for the $x_{r 1} x_{r 2}$ surface shear are numerically determined:

$$
\vec{F}_{r A+}=-\vec{F}_{r A-}, \vec{F}_{r B+}=-\vec{F}_{r B-}, \vec{F}_{r C+}=\vec{F}_{r C-}=\overrightarrow{0} .
$$

The average shear stresses on the side surfaces are:

$$
\bar{\tau}_{r 12}=\frac{F_{r 1 B+}}{A_{B+}}, \quad \bar{\tau}_{r 21}=\frac{F_{r 2 A+}}{A_{A+}} .
$$

Numerical verification opportunity of results is to check that the shear stresses should be the same on the side surfaces perpendicular to $x_{r 1}, x_{r 2}$ axes (due to the duality of $\tau$ stresses):

$$
\bar{\tau}_{r 12}=\bar{\tau}_{r 21} \text {. }
$$

The shear stress distribution is never homogenous on the roving model-cell. Figure 12 shows the $\tau_{r 12}$ stress distribution on the roving model-cell. The deformation is illustrated in a zoom of hundred times. The $X Y Z$ coordinate system in figure is identical to the $x_{r 1} x_{r 2} x_{r 3}$ coordinate system.

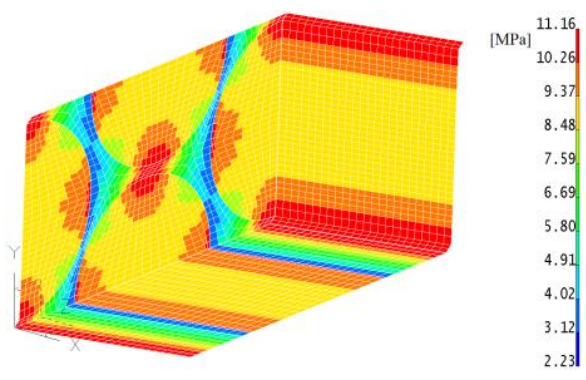

Figure 12

$\tau_{r 12}$ stress distribution on the roving model-cell 
The shear modulus on the $x_{r 1} x_{r 2}$ surface is:

$G_{r 12}=\frac{\bar{\tau}_{r 12}}{\bar{\gamma}_{r 12}}$

In order to determine the shear modulus $G_{r 23}$ of the roving material we need to model pure shear on the $x_{r 2} x_{r 3}$ surface:

$\frac{1}{2} \bar{\gamma}_{r 23}=\frac{1}{2} \bar{\gamma}_{r 32}, \quad \bar{\gamma}_{r 23}=\frac{1}{2} \bar{\gamma}_{r 23}+\frac{1}{2} \bar{\gamma}_{r 32}$.

At the shear on $x_{r 2} x_{r 3}$ plane the $A+$ and $A$ - side surfaces remain planes and do not displace in $x_{r 1}$ direction. For simulation of pure shear with the roving model-cell we have given values for the nodes at the center points of the side surfaces, as follows:

$$
\begin{array}{ll}
w_{B-}=w_{N 12}=0, & w_{B+}=w_{N 11}=a \frac{1}{2} \bar{\gamma}_{r 32}, \\
v_{C-}=v_{N 14}=0, & v_{C+}=v_{N 14}=b \frac{1}{2} \bar{\gamma}_{r 23}, \\
u_{A-}=u_{N 10}=0, & u_{A+}=u_{N 9}=0 .
\end{array}
$$

The nodes are coupled at the side surfaces with (50) - (52) relations.

$$
\begin{array}{ll}
w_{B-}=w\left(x_{r 1} ; 0 ; x_{r 3}\right)=w_{N 5}, & w_{B+}=w\left(x_{r 1} ; a ; x_{r 3}\right)=w_{N 8}, \\
v_{C-}=v\left(x_{r 1} ; x_{r 2} ; 0\right)=v_{N 3}, & v_{C+}=v\left(x_{r 1} ; x_{r 2} ; b\right)=v_{N 7}, \\
u_{A-}=u\left(0 ; x_{r 2} ; x_{r 3}\right)=u_{N 1}, & u_{A+}=u\left(a ; x_{r 2} ; x_{r 3}\right)=u_{N 2} .
\end{array}
$$

In (50) - (52) relations:

$0 \leq x_{r 1} \leq a, \quad 0 \leq x_{r 2} \leq a, \quad 0 \leq x_{r 3} \leq b$.

According to the (54) - (56) requirements the nodes have equal displacements on the opposite side surfaces, with exception to the edges of the model-cell:

$$
\begin{aligned}
& B-/ B+: \quad u\left(x_{r 1} ; 0 ; x_{r 3}\right)=u\left(x_{r 1} ; a ; x_{r 3}\right), \quad v\left(x_{r 1} ; 0 ; x_{r 3}\right)=v\left(x_{r 1} ; a ; x_{r 3}\right), \\
& C-/ C+: \quad u\left(x_{r 1} ; x_{r 2} ; 0\right)=u\left(x_{r 1} ; x_{r 2} ; b\right), \quad w\left(x_{r 1} ; x_{r 2} ; 0\right)=w\left(x_{r 1} ; x_{r 2} ; b\right), \\
& A-/ A+: \quad v\left(0 ; x_{r 2} ; x_{r 3}\right)=v\left(a ; x_{r 2} ; x_{r 3}\right), \quad w\left(0 ; x_{r 2} ; x_{r 3}\right)=w\left(a ; x_{r 2} ; x_{r 3}\right) .
\end{aligned}
$$

In relations $(54)-(56)$ and $(58)-(60)$ :

$$
0<x_{r 1}<a, \quad 0<x_{r 2}<a, \quad 0<x_{r 3}<b .
$$


For the node pairs at the opposite edges, except the corner points of the modelcell, we prescribe the relations as follows:

For edges in $x_{r 1}$ direction:

$$
u\left(x_{r 1} ; 0 ; 0\right)=u\left(x_{r 1} ; a ; 0\right)=u\left(x_{r 1} ; a ; b\right)=u\left(x_{r 1} ; 0 ; b\right),
$$

For edges in $x_{r 2}$ direction:

$$
w\left(0 ; x_{r 2} ; 0\right)=w\left(0 ; x_{r 2} ; b\right)=w\left(a ; x_{r 2} ; b\right)=w\left(a ; x_{r 2} ; 0\right),
$$

For edges in $x_{r 3}$ direction:

$$
v\left(0 ; 0 ; x_{r 3}\right)=v\left(a ; 0 ; x_{r 3}\right)=v\left(a ; a ; x_{r 3}\right)=v\left(0 ; a ; x_{r 3}\right) \text {. }
$$

With FE computation we got at the $x_{r 2} x_{r 3}$ surface shear the following reaction forces:

$$
\vec{F}_{r B+}=-\vec{F}_{r B-}, \vec{F}_{r C+}=-\vec{F}_{r C-}, \vec{F}_{r A+}=\vec{F}_{r A-}=\overrightarrow{0} .
$$

The average shear stresses on the side surfaces:

$\bar{\tau}_{r 23}=\frac{F_{r 2 C+}}{A_{C+}}, \quad \bar{\tau}_{r 32}=\frac{F_{r 3 B+}}{A_{B+}}$.

The duality of $\tau$ stresses should be valid on the side surfaces perpendicular to $x_{r 2}, x_{r 3}$ axes:

$\bar{\tau}_{r 23}=\bar{\tau}_{r 32}$.

Shear modulus on $x_{r 2} x_{r 3}$ surface:

$$
G_{r 23}=\frac{\bar{\tau}_{r 23}}{\bar{\gamma}_{r 23}}
$$

Table 4 summarizes a numerical example for comparison with the further experiments, the results of computations received as results of simulation of the roving model-cell. The table contains the given kinematic loadings for both, shear cases, the computed reaction forces, the calculated average shear stresses and the shear moduli. 
Table 4

The prescribed and defined properties at the examined roving model cell

\begin{tabular}{|l|l|}
\hline \multicolumn{2}{|c|}{ Pure shear on $x_{r 1} x_{r 2}$ surface } \\
\hline \multirow{4}{*}{ Kinematic loading } & $\bar{\gamma}_{r 12}=2 \cdot 10^{-3}, \frac{1}{2} \bar{\gamma}_{r 12}=\frac{1}{2} \bar{\gamma}_{r 21}=10^{-3}$ \\
\cline { 2 - 3 } & $v_{A+}=v_{N 9}=7.613 \cdot 10^{-3} \mathrm{~mm}, u_{B+}=u_{N 11}=7.613 \cdot 10^{-3} \mathrm{~mm}$ \\
\hline \multirow{3}{*}{ Reaction forces } & $\vec{F}_{r A+}=\left(825.9 \vec{e}_{r 2}\right) \mathrm{N}, \vec{F}_{r A-}=\left(-825.9 \vec{e}_{r 2}\right) \mathrm{N}$ \\
\cline { 2 - 3 } & $\vec{F}_{r B+}=\left(825.9 \vec{e}_{r 1}\right) \mathrm{N}, \vec{F}_{r B-}=\left(-825.9 \vec{e}_{r 1}\right) \mathrm{N}$ \\
\hline Average stresses & $\bar{\tau}_{r 12}=8.23 \mathrm{MPa}, \bar{\tau}_{r 21}=8.23 \mathrm{MPa}$ \\
\hline \multirow{2}{*}{ Shear modulus } & $G_{r 12}=4115 \mathrm{MPa}$ \\
\hline \multirow{3}{*}{ Kinematic loading } & \multicolumn{1}{|c|}{ Pure shear on $x_{r 2} x_{r 3}$ surface } \\
\cline { 2 - 3 } & $\bar{\gamma}_{r 23}=2 \cdot 10^{-3}, \frac{1}{2} \bar{\gamma}_{r 23}=\frac{1}{2} \bar{\gamma}_{r 32}=10^{-3}$ \\
\hline \multirow{3}{*}{ Reaction forces } & $w_{B+}=w_{N 11}=7.613 \cdot 10^{-3} \mathrm{~mm}, v_{C+}=v_{N 14}=13.186 \cdot 10^{-3} \mathrm{~mm}$ \\
\hline \multirow{2}{*}{ Average stresses } & $\vec{F}_{r B+}=\left(726.45 \vec{e}_{r 3}\right) \mathrm{N}, \vec{F}_{r B-}=\left(-726.45 \vec{e}_{r 3}\right) \mathrm{N}$ \\
\cline { 2 - 3 } & $\vec{F}_{r C+}=\left(419.419 \vec{e}_{r 2}\right) \mathrm{N}, \vec{F}_{r C-}=\left(-419.419 \vec{e}_{r 2}\right) \mathrm{N}$ \\
\hline Shear modulus & $\bar{\tau}_{r 23}=7.24 \mathrm{MPa}, \bar{\tau}_{r 32}=7.24 \mathrm{MPa}$ \\
\hline
\end{tabular}

\subsection{Characteristic Data of the Textile Composite Layer used in the Experiment}

The composite plate is reinforced with a SIGRATEX KDL 8003 type plain weave carbon fabric and the matrix is a polyester resin.

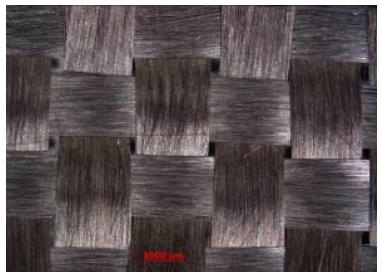

Figure 13

Plain weave fabric

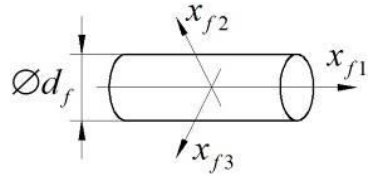

Figure 14

Material coordinate system of a carbon fiber

In Figure 13 the plain weave fabric before impregnation is seen. The fibers are ordered into flat, untwisted roving. The type of the roving is Torayca T300-3K. 
$3 \mathrm{~K}$ means that the number of fibers is $n_{f}=3000$ pieces and the diameter of a carbon fiber is $d_{f}=7 \mu \mathrm{m}$ in the roving.

Material properties of the carbon fiber originate from literature [4], [10], in the $x_{f 1} x_{f 2} x_{f 3}$ material coordinate system (Figure 14):

$$
\begin{array}{lll}
E_{f 1}=230000 \mathrm{MPa} & v_{f 12}=v_{f 13}=0.166 & G_{f 12}=G_{f 13}=6432 \mathrm{MPa} \\
E_{f 2}=E_{f 3}=15000 \mathrm{MPa} & v_{f 23}=0.400 & G_{f 23}=5357 \mathrm{MPa}
\end{array}
$$

The matrix is an AROPOL M105TB type polyester resin which is a linear-elastic, isotropic material. The Young's modulus $E_{m}$ and the Poisson's ratio $v_{m}$ were measured according to standard [1], the shear modulus $G_{m}$ was defined by the (64) relationship, valid for the isotropic materials:

$$
E_{m}=3677 \mathrm{MPa}, \quad v_{m}=0.346, \quad G_{m}=\frac{E_{m}}{2\left(1+v_{m}\right)}=1365.9 \mathrm{MPa} .
$$

\subsection{Material Properties Determined by the Roving Model-Cell}

For the investigated roving material, considering the relationships (8), we determined the material properties with the finite element roving model-cell as follows:

$$
\begin{array}{lll}
E_{r 1}=177236 \mathrm{MPa}, & v_{r 12}=v_{r 13}=0.202, & G_{r 12}=G_{r 13}=4115 \mathrm{MPa}, \\
E_{r 2}=E_{r 3}=10352 \mathrm{MPa}, & v_{r 23}=0.430, & G_{r 23}=3620 \mathrm{MPa} .
\end{array}
$$

If the reinforcing fiber in the roving is transversely isotropic, the roving has the same material behavior because the fibers are ordered hexagonally in that. Transversely isotropic materials have a plane with isotropic behavior. The given carbon fibers are isotropic in the plane of their cross sections because $G_{f 23}$ shear modulus can be determined with formula (65) which is valid for the isotropic materials:

$$
G_{f 23}=\frac{E_{f 2}}{2\left(1+v_{f 23}\right)}=\frac{15000}{2(1+0.4)}=5357 \mathrm{MPa} .
$$

The roving model-cell is also transversely isotropic because one can calculate the shear modulus $G_{r 23}=3620 \mathrm{MPa}$ determined with the model-cell, by (66) relationship:

$$
G_{r 23}=\frac{E_{r 2}}{2\left(1+v_{r 23}\right)}=\frac{10352}{2(1+0.43)}=3620 \mathrm{MPa} .
$$

The roving model-cell delivers transversely isotropic results, so the material has only five independent material constants: $E_{r 1}, E_{r 2}, v_{r 12}, v_{r 23}, G_{r 12}$. 


\section{Conclusions}

This work presents a FE roving model-cell, for determination of macroscopic, orthotropic material properties of the reinforcing roving impregnated by the matrix material. It defines the structure of the finite element roving model-cell and gives the boundary conditions applied to the finite element computations. Using this roving model-cell, we determine the material properties for a roving, where the shape of the cross sections of the roving, the number of reinforcing fibers in the roving and the material properties of the fiber and the matrix are known. It computes the macroscopic material properties of an actual roving by numerical simulation for two axial tensile tests and for two pure shear tests.

Future work will summarize the numerical determination of the material properties of a textile composite layer. The macroscopic material properties of a textile composite layer will be determined by a finite element model-cell and we will compare them with experimental results. The applied roving of the textile composite layer is the same as dealt with in this current work. Here we have carried out a numerical verification with the roving model-cell. The experimental verification will take place, by the measurement data of the layer, in the future work.

\section{References}

[1] ASTM D638-10, Standard Test Method for Tensile Properties of Plastics, 2010

[2] Bunsell, A. R.; Renard, J.: Fundamentals of Fibre Reinforced Composite Materials, Series in Material Science and Engineering, Institute of Physics Publishing, 398 p., 2005

[3] Égert J., Pere B.: Finite Element Analysis, MSc Lecture notes, UniversitasGyőr Non-profit Ltd., Győr, 218 p., 2011 (in Hungarian)

[4] Goda T.: Wear Mechanism of Composite-Steel Sliding Pairs, PhD Thesis, 2002, Budapest University of Technology and Economics, Faculty of Mechanical Engineering, Supervisor: Váradi K. (In Hungarian)

[5] Hallal, A., Fardoun, F., Younes, R., Hage Chehade, F.: Evaluation of Longitudinal and Transversal Young's Moduli for Unidirectional Composite Material with Long Fibers, Advanced Materials Research, Vol. 324, p. 189192, 2011

[6] Jones, R. M.: Mechanics of Composite Materials, Second Edition, Taylor \& Francis Group, 519 p., 1999

[7] Leijan H.: Determining Micro- and Macro- Geometry of Fabric and Fabric Reinforced Composites, Dissertation, 2013, Department of Mechanical and Nuclear Engineering College of Engineering, Kansas State University, Manhattan, Kansas, Advisor: Youqi W.

[8] Siva Bhaskara Rao Devireddy; Sandhyarani Biswas: Effect of Fiber Geometry and Representative Volume Element on Elastic and Thermal Properties 
of Unidirectional Fiber-Reinforced Composites, Hindawi Publishing Corporation Journal of Composites, Article ID 629175, 12 pages, 2014

[9] Srinivasa, V., Shivakumar, V., Nayaka, V., Jagadeeshaiaih, S., Seethram, M., Shenoy, R., Nafidi, A.: Fracture Morphology of Carbon Fiber Reinforced Plastic Composite Laminates, Materials Research, Vol. 13, No. 3, pp. $417-424,2010$

[10] Torayca T300 factory data sheet catalogue (No. CFA-001) Toray Carbon Fibers America, Inc. (CFA), 2012

[11] Xia, Z., Zhang, Y., Ellyin, F.: A Unified Periodical Boundary Conditions for Representative Volume Elements of Composites and Applications, International Journal of Solids and Structures, Vol. 40, No. 8, p. 1907-1921, 2003

[12] Zhang., C.: Multi-Scale Characterization and Failure Modeling of Carbon/Epoxy Triaxially Braided Composite, Dissertation, 2013, The Graduate Faculty of The University of Akron, Advisor: Binienda, W. K 